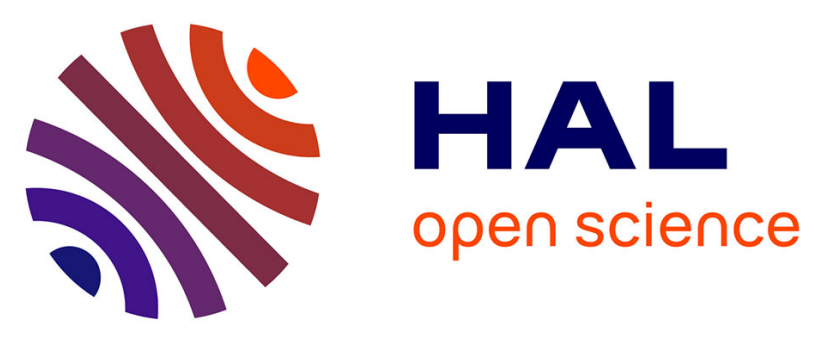

\title{
Les relations internationales
}

Dario Battistella

\section{To cite this version:}

Dario Battistella. Les relations internationales. Sciences humaines, 2007, 183, pp.52-56. halshs00155040

\section{HAL Id: halshs-00155040 https://shs.hal.science/halshs-00155040}

Submitted on 15 Jun 2007

HAL is a multi-disciplinary open access archive for the deposit and dissemination of scientific research documents, whether they are published or not. The documents may come from teaching and research institutions in France or abroad, or from public or private research centers.
L'archive ouverte pluridisciplinaire HAL, est destinée au dépôt et à la diffusion de documents scientifiques de niveau recherche, publiés ou non, émanant des établissements d'enseignement et de recherche français ou étrangers, des laboratoires publics ou privés. 
pour $\underline{\text { Sciences Humaines }}$ Rubrique « Courants et disciplines »

\section{Les Relations internationales}

La discipline Relations internationale n'a pas un siècle d'existence. Issue de la science politique elle s'est constituée dans un but noble, favoriser la paix, avant de privilégier des objectifs de connaissance. Peu à peu, elle a intégré des théories et des éléments d'analyse issus d'autres sciences sociales. On peut y voir un danger de dissolution. Mais c'est aussi le signe d'une grande vitalité de la part d'une discipline essentielle pour comprendre le monde.

Les Relations internationales (RI) sont officiellement nées en 1919, lorsque le mécène gallois David Davies finança la création de la première chaire de politique internationale à l'University College of Wales à Aberystwyth. Le contexte, l'immédiat après-Première guerre mondiale, expliquait à la fois la raison d'être, l'objet d'étude, et l'intérêt cognitif de la nouvelle discipline. Côté raison d'être, il s'agissait de procéder à une étude systématique des relations internationales, en lieu et place des réflexions parcellaires et intuitionnistes qui, de Thucydide à Marx, avaient été proposées tout au long de l'histoire de la pensée politique. L'objet d'étude se restreignait, lui, aux seules relations interétatiques concernées par la problématique de la guerre et de la paix, d'où la dénomination retenue - politique internationale. Côté objectif, les premiers internationalistes, idéalistes majoritairement britanniques, revendiquaient ouvertement les valeurs normatives qui guidaient leurs recherches : de même que la médecine avait pour but d'étudier les causes des maladies pour promouvoir la santé, de même l'étude scientifique de la politique internationale devait contribuer à favoriser les chances de la paix sur les risques de guerre.

Depuis, ce dernier objectif a été abandonné, suite notamment au démenti apporté par la Seconde guerre mondiale et la guerre froide à l'optimisme des idéalistes, convaincus de la capacité du droit international, de la démocratie et du libre-échange à stabiliser la paix. Certes, dans la lignée de l'Ecole de Francfort, le post-positiviste canadien Robert Cox a récemment rappelé qu'en RI en tant que science sociale, toute théorie est toujours pour quelqu'un et pour quelque chose, que le théoricien en soit conscient ou non. Mais pour la grande majorité des internationalistes contemporains, il est possible de proposer des connaissances savantes sur les relations internationales : pour être chargées de théorie, celles-ci ne sont pas pour autant déterminées par des valeurs. Il reste que ce quasi-consensus épistémologique n'a mis fin ni aux débats relatifs à la définition de l'objet d'étude des RI, ni aux controverses portant sur les méthodes les plus pertinentes pour aborder cet objet. Tout au contraire, la discipline scientifique RI est aussi un champ savant, caractérisé par des débats sans fin, sinon des dialogues de sourds, entre paradigmes concurrents.

\section{Au commencement était l'anarchie}

Pendant les cinquante premières années de son existence, la question de la délimitation des RI n'a guère posé de problème. Dans la continuité des philosophes du contrat (Hobbes, Locke, Rousseau, ...), qui établissent une séparation nette entre l'ordre interne hiérarchique, avec une autorité centrale revendiquant le monopole de la violence physique légitime, et la sphère internationale anarchique, dépourvue d'une telle instance, les premiers internationalistes sont anarchophiles: ils partent du postulat que l'anarchie, au sens étymologique d'absence d'autorité supérieure, est tout à la fois le trait fondamental de la vie internationale et le point de départ de toute réflexion théorique sur celle-ci. A ce premier postulat s'en ajoute un second, le stato-centrisme : seuls sont reconnus comme acteurs des relations internationales 
les Etats, entités souveraines qui, depuis les Traités de Westphalie en 1648, sont devenues la forme d'organisation privilégiée des sociétés humaines; par ailleurs, ces acteurs étatiques sont considérés comme des acteurs unitaires et rationnels incarnés par les détenteurs du pouvoir exécutif.

C'est de cette période de l'entre-deux guerres que date le principal axe de structuration de la discipline, autour de l'opposition entre les idéalistes, qui constituent la première variante du libéralisme en RI, et les réalistes. Ces deux approches s'intéressent essentiellement aux questions de la guerre et de la paix ou, pour employer des termes plus modernes, des conflits et de la coopération, tout en divergeant sur l'interprétation à donner de l'anarchie : pour les réalistes, celle-ci est synonyme d'état de guerre éternel, donnée objective qui contraint les Etats à ne compter que sur soi et à se comporter selon les canons de la politique de puissance ; pour les libéraux au contraire, l'anarchie est une variable évolutive, susceptible de déboucher progressivement sur une résolution des conflits par la coopération plutôt que par le recours à la force. Depuis l'opposition fondatrice entre Edward Carr et Hans Morgenthau d'un côté, et les idéalistes britanniques de l'entre-deux guerres de l'autre, ce débat parcourt toutes les générations d'internationalistes, jusqu'à la distinction contemporaine établie par Joseph Nye entre hard power (puissance de contrainte, de coercition) et soft power (puissance de séduction, de cooptation), et avec pour point d'orgue le débat opposant dans les années 19801990 les néoréalistes structuralistes aux néolibéraux institutionnalistes américains au sujet des régimes internationaux. Le constructivisme soft d'Alexander Wendt s'inscrit lui aussi dans cet axe : bien que reposant sur des postulats ontologiques différents qui l'amènent à distinguer trois types de structures anarchiques culturelles - hobbienne, lockienne, kantienne - plutôt qu'un type de structure anarchique matérielle, l'analyse de Wendt porte bien sur l'étude de la violence internationale et de sa régulation, comme l'indique le titre de son livre - Social Theory of International Politics -, allusion directe à la bible néo-réaliste qu'est l'ouvrage Theory of International Politics de Kenneth Waltz.

Ceci étant, si le noyau dur des RI continue de nos jours d'attirer de nombreux internationalistes, il ne fait plus l'unanimité. Plus précisément, le stato-centrisme d'abord, la primauté des seuls enjeux de sécurité de l'autre, ont été remis en cause.

\section{Transnationalisme et économie politique internationale}

Dès la fin des années soixante, les premières brèches commencent à lézarder l'édifice du stato-centrisme, sous les coups de boutoir des néo-marxistes d'un côté et des transnationalistes de l'autre. Pour les néo-marxistes, des dependendistas à Immanuel Wallerstein sans oublier Johan Galtung, les acteurs des relations internationales ne sont pas principalement les Etats, mais les classes, l'Etat n'étant qu'une superstructure au service des intérêts de la classe dominante. Les intérêts en question sont en dernière analyse de nature économique plutôt que politique; quant au système international, il est d'abord un marché mondial dont la structure n'est pas déterminée par les rapports de puissance, mais par les rapports de production et d'échange entre le centre et la périphérie. Pour les transnationalistes, l'Etat n'est qu'un acteur parmi d'autres, à côté des acteurs sociétaux ou non-gouvernementaux que sont les entreprises multinationales, les ONG de toutes sortes, voire les individus. Entre ces acteurs non-étatiques se nouent des relations transnationales qui viennent à la fois concurrencer et compléter les relations interétatiques stricto sensu. En effet, dans la mesure où celles-ci se construisent directement dans l'espace mondial en échappant au moins partiellement au contrôle et à l'action médiatrice des Etats, elles provoquent une interdépendance complexe, pour reprendre l'expression de Robert Keohane et Joseph Nye, voire un monde mixte composé d'une sphère interétatique et d'une sphère multicentrée, comme le dit James Rosenau.

Keohane et Nye, ainsi que Rosenau, sont, à une génération d'intervalle, les principaux représentants du transnationalisme américain. Mais c'est surtout en Europe que le 
transnationalisme a pris racine, en Grande Bretagne grâce à John Burton et son concept de société mondiale ; en France lorsque Marcel Merle s'oppose au réalisme de Raymond Aron dans les années soixante-dix, et grâce à Bertrand Badie et Marie-Claude Smouts dans les années quatre-vingt-dix. Aux Etats-Unis, le succès de la contre-attaque néo-réaliste orchestrée par Kenneth Waltz explique le statut in fine marginal du transnationalisme, pour cause de triomphe du débat néo-néo auquel Keohane a d'ailleurs été le premier à participer en tant que principal représentant du néo-libéralisme institutionnel.

En revanche, si le stato-centrisme n'a guère perdu de sa splendeur aux Etats-Unis, sauf récemment chez quelques libéraux pluralistes redécouvrant, tels Andrew Moravcsik, l'individu rationnel comme acteur premier, la focalisation sur les problèmes de sécurité n'est quant à elle plus du tout ce qu'elle était. Témoins de la fin du système de Bretton Woods et de la crise pétrolière, certains théoriciens y compris réalistes tels que Robert Gilpin, commencent à s'intéresser aux dimensions économiques des relations internationales au cours des années 70-80. Aiguillés par la Britannique Susan Strange, ils fondent l'économie politique internationale, dont le but est l'étude des interactions de l'économique et du politique dans l'arène mondiale. Le succès de ce nouveau domaine de recherche est tel qu'aux Etats-Unis, de nos jours, la discipline est de facto divisée en deux sous-domaines : celui de l'International Security d'un côté, celui de l'International Political Economy de l'autre, ce dernier regroupant plus ou moins tout ce qui n'appartient pas au noyau dur classique de la politique internationale.

L'impact de cette diversification substantielle des RI sur la discipline est, dans l'ensemble, ambigu. Dans une certaine mesure, l'on assiste à un enrichissement des études internationales, étant donné que ces nouvelles approches viennent compléter plus que concurrencer les interrogations classiques, dont les analyses relatives aux enjeux étatiques ne sont guère remises en cause. Il n'empêche, mettre l'accent sur les relations transnationales, le rôle des acteurs sociétaux, souligner la transformation de l'économie politique internationale en économie politique globale avec les problèmes de gouvernance que celle-ci soulève, n'est pas sans conséquence sur le postulat de base de la discipline. La structure anarchique de la scène internationale et son corollaire le principe de stricte séparation interne-externe perdent de leur pertinence. A force de parler de globalisation des relations internationales, on finit par diluer l'identité de la discipline. En soi, définir les RI comme l'étude de la structuration de l'espace mondial par l'ensemble des réseaux d'interactions sociales n'est nullement illégitime, au vu ne serait-ce que des angles morts des RI tels que les phénomènes du terrorisme, des guerres civiles, du retour de la culture et notamment du fait religieux. Reste à savoir si un tel glissement vers les global studies est compatible avec la science politique, dont relèvent les RI en tant que sous-discipline, ou s'il signifie un passage avec armes et bagages à la sociologie, dont se réclame la plupart des globalistes, depuis le précurseur Anthony Giddens à ses représentants contemporains tels que Arjun Appadurai, Ulrich Beck, Manuel Castells et bien d'autres.

Dans tous les cas, une telle transformation exigerait une clarification d'importantes questions épistémologiques et méthodologiques qui, à notre connaissance, n'a pas eu lieu. En attendant, il ne fait guère de doute que dans l'histoire des RI de tels débats épistémologiques ont déjà eu lieu et, mieux, ils ont constitué le deuxième axe de structuration de la discipline.

\section{Du behavourisme au post-positivisme}

Lorsque la discipline fut créée, l'inspiration qu'elle subit de la part de la pensée politique ne concernait pas seulement les postulats de fond. Elle concernait aussi la méthode : les premiers internationalistes revendiquaient une démarche théorique dérivant de la philosophie, de l'histoire et du droit, et accordant une confiance explicite au jugement éthique et à la perception scientifiquement imparfaite. Cette conception dite traditionnelle fut critiquée aux Etats-Unis suite à la révolution behaviouriste des années cinquante-soixante: convaincus 
que les mêmes méthodes qui avaient permis de dévoiler les mystères de la structure atomique pouvaient révéler la dynamique du comportement international, les adeptes de la méthode dite scientifique rejetèrent l'ambition des grandes théories au profit de la minutie de la vérification empirique et de la conceptualisation opératoire des théories à moyenne portée. Ils se proposèrent de remplacer les affirmations intuitives des classiques ainsi que leur utilisation anecdotique de l'histoire par des raisonnements et des concepts rigoureux et systématiques, favorisérent la formalisation et la quantification que facilitait l'invention de l'ordinateur, et ouvrirent les RI aux statistiques, aux sciences économiques, à la théorie des jeux, à la théorie des communications, etc.

Après avoir provoqué une passe d'armes mémorable entre Morton Kaplan et Hedley Bull, ce premier débat méthodologique s'est conclu par une paix des braves: les traditionalistes reconnaissent la valeur de l'apport des behaviouristes, y compris de nature substantielle comme dans le cas de la notion de communauté de sécurité de Karl Deutsch ; quant aux behaviouristes, ils admettent qu'il est illusoire de vouloir se contenter de ne réunir que des données objectives, étant donné que les relations internationales respirent la politique et l'éthique. En fait, c'est dans les revues savantes que l'on retrouve la manifestation la plus visible de la division entre partisans de la méthode traditionnelle et adeptes des méthodes quantitatives et formelles : certaines revues, essentiellement américaines - International Studies Quarterly, Journal of Conflict Resolution -, ne publient que des articles mobilisant toutes sortes d'équations mathématiques; d'autres au contraire sont rétives à la moindre formalisation quantitative - International Security, Review of International Studies, European Journal of International Relations - ; d'autres encore sont plus œcuméniques, à l'image de International Organization ou du Journal of Peace Research.

Cette cohabitation s'explique également par l'émergence d'un nouveau débat. Les traditionalistes et behaviouristes ont beau être opposés au sujet de la meilleure méthode scientifique, ils n'en sont pas moins d'accord pour partager la même conception positive, ou positiviste, au sens d'Auguste Comte, de la faisabilité des sciences sociales. Plus précisément, ils sont d'accord pour dire que l'on peut distinguer les faits et les valeurs, pour postuler qu'il existe des régularités causales au sein du monde social susceptibles d'être découvertes par la recherche scientifique, pour affirmer qu'il est possible de vérifier empiriquement la validité des explications données de ces régularités. Ce sont ces certitudes qui ont été ébranlées dans les années 80-90, au moment du débat épistémologique opposant positivistes et postpositivistes. D'après les théoriciens critiques inspirés par Gramsci et l'Ecole de Francfort, les post-modernistes influencés par Foucauld et Derrida, ainsi que les féministes, il ne saurait y avoir de connaissance objective des relations sociales et donc internationales, et ce à cause de la nature socialement construite à la fois des théories scientifiques et des réalités sociales. Les connaissances produites ne sont jamais que des discours partisans subjectifs, et à ce titre ne sont ni réfutables, ni cumulables, ni même communicables, chaque approche ayant son propre régime de vérité.

Les critiques soulevées par les constructivistes hard ont été ignorées par les approches mainstream, largement insensibles à tout débat métathéorique en RI. Mais elles ont été prises au sérieux par les constructivistes soft qui, désireux de proposer une synthèse, ont refusé de jeter le bébé scientifique avec l'eau du bain positiviste. Associé à Alexander Wendt, le constructivisme moderniste se propose de combiner l'épistémologie positiviste - la réalité sociale est susceptible de faire l'objet d'une étude savante - avec une ontologie postpositiviste - la réalité internationale n'est ni objective, ni subjective, mais intersubjective, c'est-à-dire qu'elle est ce que les agents, eux-mêmes constitués par la structure des significations partagées tout en co-constituant celle-ci, sont d'accord pour dire qu'elle est. Son succès est tel depuis une dizaine d'années qu'il en est venu à constituer le troisième pilier de la discipline, à côté du réalisme et du libéralisme : publié en 1992, l'article Anarchy Is 
What States Make of It est la publication la plus citée de l'ensemble de la discipline depuis sa parution. Si ce succès confirme la tendance des RI à former un ménage à trois - le constructivisme a remplacé le marxisme -, il met également un terme définitif à tout espoir de faire des RI une science normale, au sens de Thomas Kuhn. Mais il n'est pas sûr qu'il faille déplorer cet état de fait car, après tout, il confirme le pronostic émis en son temps par Max Weber au sujet des sciences sociales : les RI sont une science à laquelle il est donné de rester éternellement jeune, vu que le flux perpétuellement mouvant de la civilisation lui procure sans cesse de nouveaux problèmes à étudier.

\section{Encadré 'Grands auteurs'}

Edward Carr (1892-1982)

L'historien britannique a été plus qu'un simple précurseur du réalisme. Sa critique de l'idéalisme de l'entre-deux-guerres a aussi posé les bases des approches critiques des RI en voyant dans la praxis des puissances dominantes une rationalisation de leurs intérêts.

\section{Hans Morgenthau (1904-1980)}

Elève de Max Weber avant de s'enfuir aux Etats-Unis, le fondateur du réalisme classique américain est à l'origine de l'envol de la discipline aux Etats-Unis, et ipso facto dans le monde.

Raymond Aron (1905-1983)

Partisan d'une approche sociologique, id est compréhensive plutôt qu'explicative, des RI, Aron est un réaliste atypique. Il est le seul français pouvant se targuer d'un dialogue scientifique transatlantique, successivement avec H. Morgenthau et K. Waltz.

\section{Hedley Bull (1932-1985)}

Farouche opposant à l'introduction des méthodes behaviouristes en RI, le principal représentant de l'Ecole anglaise est également à l'origine du renouveau du libéralisme statocentré moderne, voire du constructivisme soft d'un A. Wendt.

Kenneth Waltz (né en 1924)

Pape du néo-réalisme américain, Waltz est la figure incontournable de la discipline contemporaine. Sa Theory of International Politics a structuré les débats à la fois substantiels et épistémologiques de ces quelques trente dernières années.

Robert Keohane (né en 1941)

Ce touche-à-tout a été partie prenante à l'ensemble des débats inter-paradigmatiques depuis les années soixante-dix, grâce aussi à son positionnement dans le champ des RI américaines : transnationalisme $v s$. stato-centrisme, néo-libéralisme institutionnaliste $v s$. néo-réalisme structuraliste, approches mainstream vs. approches post-positivistes.

Susan Strange (1923-1998)

L'économie politique internationale, l'une des branches les plus prometteuses des RI contemporaines, doit son existence à la franc-tireure britannique, pourfendeure infatigable de toutes les langues de bois savantes. 
James Rosenau (né en 1924)

Behaviouriste dans les années soixante, Rosenau est devenu le principal représentant américain de l'approche transnationaliste depuis la fin de la guerre froide. Marginalisé chez lui mais apprécié en France, il a diagnostiqué la dialectique de la mondialisation, incarnée dans les deux figures que sont le touriste et le terroriste.

Alexander Wendt (né en 1958)

Dans une discipline verrouillée par le débat «néo-néo » (néo-réalisme vs. néo-libéralisme), cet américain d'origine allemande est parvenu à faire du constructivisme, jusque là marginal voire stérile, la troisième approche principale de la discipline, en lieu et place du néomarxisme.

\section{Encadré '« Retour de l'état de guerre » ou de l'utilité des RI'}

A quoi bon les RI ? Cette question, teintée d'un scepticisme volontiers moqueur, est souvent posée à une discipline incapable de prévoir la fin de la guerre froide ou les attentats du 11 septembre, et inapte à empêcher la guerre américaine contre l'Irak pourtant critiquée par toute la profession, y compris aux Etats-Unis. En fait, la première fonction des RI, comme de toute discipline scientifique, consiste, à partir d'une hypothèse théorique, à décrire un événement (ou une série d'événements), et à tenter d'en donner une explication. C'est ce que montre l'ouvrage « Retour de l'état de guerre », à propos justement de l'opération Liberté en Irak.

L'analyse est fondée sur une hypothèse théorique empruntée au libéralisme stato-centré : il existe en politique internationale une tendance lourde vers l'avènement d'une société internationale, synonyme de pacification progressive des relations interétatiques, et ce depuis que le système international est stabilisé par une puissance prépondérante, la Grande Bretagne en 1815, les Etats-Unis depuis 1945.

Elle se poursuit avec une description de l'opération « Liberté en Irak » recourant aux concepts fournis par le constructivisme : en réintroduisant la figure de l'ennemi dans sa perception de l'Irak de Saddam Hussein, en réhabilitant la doctrine de la guerre préventive, en agissant unilatéralement, " Liberté en Irak » s'inscrit en rupture par rapport à cette tendance lourde, car elle signifie le retour de valeurs hobbiennes au détriment de la culture anarchique lockienne caractéristique de la société internationale contemporaine.

Elle se conclut par une tentative d'explication du comportement américain et, pour ce faire, se positionne de façon critique aux différents niveaux d'analyse que fournissent les théories libérales et réalistes de la guerre et de la paix en RI. La thèse retenue est la suivante : si on peut difficilement voir dans "Liberté en Irak » une conséquence du dilemme de la sécurité qu'auraient ressenti les Etats-Unis de la part de l'Irak, on peut, en revanche, y voir à la fois une guerre impérialiste, dans la mesure où il s'agit pour certains groupes sociétaux américains de promouvoir leurs intérêts mesquins, et une guerre proto-systémique, étant donné que le comportement américain renvoie à la nécessité pour Washington de gérer son déclin annoncé face à l'ascension du futur peer competitor pour l'hégémonie mondiale qu'est, à ses yeux, la Chine. 